\title{
Vertex degree sums for matchings in 3-uniform hypergraphs
}

\author{
Yi Zhang * \\ School of Sciences \\ Beijing University of Posts and Telecommunications \\ Beijing, China \\ shouwangmm@sina.com \\ Yi Zhao ${ }^{\dagger}$ \\ Department of Mathematics and Statistics \\ Georgia State University \\ Atlanta, U.S.A. \\ yzhao6@gsu.edu
Mei $\mathrm{Lu}^{\ddagger}$
Department of Mathematical Sciences
Tsinghua University
Beijing, China
mlu@math.tsinghua.edu.cn

Submitted: Mar 30, 2019; Accepted: Sep 16, 2019; Published: Oct 11, 2019

(c) The authors. Released under the CC BY-ND license (International 4.0).

\begin{abstract}
Let $n, s$ be positive integers such that $n$ is sufficiently large and $s \leqslant n / 3$. Suppose $H$ is a 3 -uniform hypergraph of order $n$ without isolated vertices. If $\operatorname{deg}(u)+$ $\operatorname{deg}(v)>2(s-1)(n-1)$ for any two vertices $u$ and $v$ that are contained in some edge of $H$, then $H$ contains a matching of size $s$. This degree sum condition is best possible and confirms a conjecture of the authors [Electron. J. Combin. 25 (3), 2018], who proved the case when $s=n / 3$.
\end{abstract}

Mathematics Subject Classifications: 05C70,05C65

\section{Introduction}

A $k$-uniform hypergraph $H$ (in short, $k$-graph) is a pair $(V, E)$, where $V$ is a finite set of vertices and $E$ is a family of $k$-element subsets of $V$. Note that a 2-graph is simply a graph. Let $V(H)$ and $E(H)$ denote the vertex set and edge set of $H$, respectively. A matching of size $s$ in $H$ is a family of $s$ pairwise disjoint edges of $H$. If the matching covers

\footnotetext{
${ }^{*}$ Supported by the National Natural Science Foundation of China (Grant 11901048).

${ }^{\dagger}$ Partially supported by NSF grant DMS-1700622.

${ }^{\ddagger}$ Supported by the National Natural Science Foundation of China (Grant 11771247).
} 
all the vertices of $H$, then we call it a perfect matching. Given a set $S \subseteq V$, the degree $\operatorname{deg}_{H}(S)$ of $S$ is the number of the edges of $H$ containing $S$. We simply write $\operatorname{deg}(S)$ when $H$ is obvious from the context. Further, let $\delta_{\ell}(H)=\min \{\operatorname{deg}(S): S \subseteq V(H),|S|=\ell\}$.

Given integers $\ell<k \leqslant n$ such that $k$ divides $n$, let $m_{\ell}(k, n)$ denote the smallest integer $m$ such that every $k$-graph $H$ on $n$ vertices with $\delta_{\ell}(H) \geqslant m$ contains a perfect matching. In recent years the problem of determining $m_{\ell}(k, n)$ has received much attention (see $[2,5,6,7,8,9,10,12,14,17,16,18,20,21,22])$. In particular, Rödl, Ruciński and Szemerédi [18] determined $m_{k-1}(k, n)$ for all $k \geqslant 3$ and sufficiently large $n$. Treglown and Zhao $[20,21]$ determined $m_{\ell}(k, n)$ for all $\ell \geqslant k / 2$ and sufficiently large $n$. More Dirac-type results on hypergraphs can be found in surveys [15, 27].

A well-known result of Ore [13] extended Dirac's theorem by determining the smallest degree sum of two non-adjacent vertices that guarantees a Hamilton cycle in graphs. Oretype problems for hypergraphs have been studied recently. For example, Tang and Yan [19] studied the degree sum of two $(k-1)$-sets that guarantees a tight Hamilton cycle in $k$-graphs. Zhang and Lu [23] studied the degree sum of two $(k-1)$-sets that guarantees a perfect matching in $k$-graphs. Zhang, Zhao and $\mathrm{Lu}$ [26] determined the minimum degree sum of two adjacent vertices that guarantees a perfect matching in 3-graphs without isolated vertices, see Theorem 2 (two vertices in a hypergraph are adjacent if there exists an edge containing both of them). Note that one may study the minimum degree sum of two arbitrary vertices and that of two non-adjacent vertices that guarantees a perfect matching instead. In fact, it was mentioned in [26] that the former equals to $2 m_{1}(3, n)-1$ while the latter does not exist.

Let us define (potential) extremal 3-graphs for the matching problem. For $1 \leqslant \ell \leqslant 3$, let $H_{n, s}^{\ell}$ denote the 3 -graph of order $n$, whose vertex set is partitioned into two sets $S$ and $T$ of size $n-s \ell+1$ and $s \ell-1$, respectively, and whose edge set consists of all triples with at least $\ell$ vertices in $T$. A well-known conjecture of Erdős [3], recently verified for 3-graphs $[4,11]$, implies that $H_{n, s}^{1}$ or $H_{n, s}^{3}$ is the densest 3-graph on $n$ vertices not containing a matching of size $s$. On the other hand, Kühn, Osthus and Treglown [10] showed that for sufficiently large $n, H_{n, s}^{1}$ has the largest minimum vertex degree among all 3-graphs on $n$ vertices not containing a matching of size $s$.

Theorem 1. [10] There exists $n_{0} \in \mathbb{N}$ such that if $H$ is a 3-graph of order $n \geqslant n_{0}$ with $\delta_{1}(H)>\delta_{1}\left(H_{n, s}^{1}\right)=\left(\begin{array}{c}n-1 \\ 2\end{array}\right)-\left(\begin{array}{c}n-s \\ 2\end{array}\right)$ and $n \geqslant 3 s$, then $H$ contains a matching of size $s$.

Given a 3-graph $H$, let $\sigma_{2}(H)$ denote the minimum $\operatorname{deg}(u)+\operatorname{deg}(v)$ among all adjacent vertices $u$ and $v$. It is easy to see that

$$
\begin{aligned}
& \sigma_{2}\left(H_{n, s}^{3}\right)=2\left(\begin{array}{c}
3 s-2 \\
2
\end{array}\right), \quad \sigma_{2}\left(H_{n, s}^{1}\right)=2\left(\left(\begin{array}{c}
n-1 \\
2
\end{array}\right)-\left(\begin{array}{c}
n-s \\
2
\end{array}\right)\right), \text { and } \\
& \sigma_{2}\left(H_{n, s}^{2}\right)=\left(\begin{array}{c}
2 s-2 \\
2
\end{array}\right)+(n-2 s+1)\left(\begin{array}{c}
2 s-2 \\
1
\end{array}\right)+\left(\begin{array}{c}
2 s-1 \\
2
\end{array}\right)=(2 s-2)(n-1) .
\end{aligned}
$$

The following is [26, Theorem 1], which implies that, when $n$ is divisible by 3 and sufficiently large, $H_{n, n / 3}^{2}$ has the largest $\sigma_{2}(H)$ among all $n$-vertex 3 -graphs $H$ containing no isolated vertex or perfect matching. 
Theorem 2. [26] There exists $n_{0} \in \mathbb{N}$ such that the following holds for all integers $n \geqslant n_{0}$ that are divisible by 3 . Let $H$ be a 3-graph of order $n$ without an isolated vertex. If $\sigma_{2}(H)>\sigma_{2}\left(H_{n, n / 3}^{2}\right)=\frac{2}{3} n^{2}-\frac{8}{3} n+2$, then $H$ contains a perfect matching.

Zhang, Zhao and Lu [26, Conjecture 12] further conjectured that for sufficiently large $n$ and any $s<n / 3, H_{n, s}^{2}$ has the largest $\sigma_{2}(H)$ among all $n$-vertex 3 -graphs $H$ containing no isolated vertex or matching of size $s$. In this paper we verify this conjecture.

Theorem 3. There exists $n_{1} \in \mathbb{N}$ such that the following holds for all integers $n \geqslant n_{1}$ and $s \leqslant n / 3$. If $H$ is a 3-graph of order $n$ without an isolated vertex and $\sigma_{2}(H)>\sigma_{2}\left(H_{n, s}^{2}\right)=$ $2(s-1)(n-1)$, then $H$ contains a matching of size $s$.

Since the two theorems have different extremal hypergraphs, Theorem 3 does not imply Theorem 1 (analogously Theorem 1 does not imply Erdős' matching conjecture for 3-graphs). On the other hand, one may wonder why we assume that $H$ contains no isolated vertex in Theorem 3 (especially when $s<n / 3$ ). In fact, as shown in the concluding remarks of [26], Theorem 3 implies another conjecture [26, Conjecture 13], which determines the largest $\sigma_{2}(H)$ among all 3-graphs containing no matching of size $s$. Note that $\sigma_{2}\left(H_{n, s}^{2}\right) \geqslant \sigma_{2}\left(H_{n, s}^{3}\right)$ if and only if $s \leqslant(2 n+4) / 9$.

Corollary 4. There exists $n_{2} \in \mathbb{N}$ such that the following holds. Suppose that $H$ is a 3 -graph of order $n \geqslant n_{2}$ and $2 \leqslant s \leqslant n / 3$. If $\sigma_{2}(H)>\max \left\{\sigma_{2}\left(H_{n, s}^{2}\right), \sigma_{2}\left(H_{n, s}^{3}\right)\right\}$, then $H$ contains a matching of size $s$.

Let us explain our approach towards Theorem 3. The case when $s \leqslant n / 13$ was already solved by Zhang and $\mathrm{Lu}[24]$ in a stronger form. Note that $\sigma_{2}\left(H_{n, s}^{2}\right)>\sigma_{2}\left(H_{n, s}^{1}\right)$. The following theorem shows that, when $n \geqslant 13 s$, not only is $H_{n, s}^{2}$ the (unique) 3-graph with the largest $\sigma_{2}(H)$ among all $H$ containing no isolated vertex or a matching of size $s$, but also $H_{n, s}^{1}$ is the sub-extremal 3-graph for this problem. (In fact, Zhang and Lu [24] conjectured that Theorem 5 holds for all $n \geqslant 3 s$. If true, this strengthens Theorem 1 and actually provides a link between Ore's and Dirac's problems.)

Theorem 5. [25] Let $n, s$ be positive integers and $H$ be a 3-graph of order $n \geqslant 13$ s without an isolated vertex. If $\sigma_{2}(H)>\sigma_{2}\left(H_{n, s}^{1}\right)=2\left(\left(\begin{array}{c}n-1 \\ 2\end{array}\right)-\left(\begin{array}{c}n-s \\ 2\end{array}\right)\right)$, then either $H$ contains a matching of size $s$ or $H$ is a subgraph of $H_{n, s}^{2}$.

Therefore it suffices to prove Theorem 3 for reasonably large $s$. For such $s$, we actually prove a (stronger) stability theorem.

Theorem 6. Given $0<\varepsilon \ll \tau \ll 1$, let $n$ be sufficiently large and $\tau n<s \leqslant n / 3$. If $H$ is a 3-graph of order $n$ without an isolated vertex such that $\sigma_{2}(H)>2 s n-\varepsilon n^{2}$, then either $H$ is a subgraph of $H_{n, s}^{2}$ or $H$ contains a matching of size $s$.

Theorem 3 follows from Theorem 6 immediately. Indeed, if $\sigma_{2}(H)>\sigma_{2}\left(H_{n, s}^{2}\right)$, then it is easy to see that $H$ is not a subgraph of $H_{n, s}^{2} \cdot{ }^{1}$ Suppose instead, that $V(H)$ can be

\footnotetext{
${ }^{1}$ Unfortunately $\sigma_{2}$ is not a monotone function: for example, adding an edge to $H_{n, s}^{2}$ indeed reduces the value of $\sigma_{2}$ because two vertices in $S$ now become adjacent and their degree sum is smaller than $\sigma_{2}\left(H_{n, s}^{2}\right)$.
} 
partitioned $S \cup T$ such that $|S|=n-2 s+1,|T|=2 s-1$, and every edge of $H$ contains at least two vertices of $T$. Since $H$ contains no isolated vertices, every vertex of $S$ is adjacent to some vertex of $T$. Thus $\sigma_{2}(H) \leqslant \operatorname{deg}(u)+\operatorname{deg}(v)$ for some $u \in S$ and $v \in T$. Consequently $\sigma_{2}(H) \leqslant \sigma_{2}\left(H_{n, s}^{2}\right)$, a contradiction. We therefore apply Theorem 6 to derive that $H$ contains a matching of size $s$. Furthermore, Theorem 6 implies that $H_{n, s}^{2}$ is the unique extremal 3-graph for Theorem 3 because all proper subgraphs $H$ of $H_{n, s}^{2}$ satisfy $\sigma_{2}(H)<\sigma_{2}\left(H_{n, s}^{2}\right)$.

In order to prove Theorem 6, we follow the same approach as in [26]: using the condition on $\sigma_{2}(H)$, we greedily extend a matching of $H$ until it has $s$ edges. An important intermediate step is finding a matching that covers a certain number of low-degree vertices (see Lemma 7). Nevertheless, the proof of Theorem 6 does require new ideas: in particular, the meaning of an optimal matching is more complicated (see Definition 8); we proceed differently depending on whether the number of low-degree vertices in the optimal matching is at the threshold. In one case we reduce the problem to that of finding a perfect matching in a subgraph of $H$ and apply the main result of [26] (see Theorem 9).

This paper is organized as follows. In Section 2, we give an outline of the proof along with some preliminary results. We prove Lemma 7 in Section 3 and complete the proof in Section 4.

Notation: Given a graph $G$ and a vertex $u$ in $G, N_{G}(u)$ is the set of neighbors of $u$ in $G$. Suppose $H$ is a 3-uniform hypergraph. For $u \neq v \in V(H)$, let $N_{H}(u, v)=\{w \in V(H)$ : $\{u, v, w\} \in E(H)\}$ (the subscript is often omitted when $H$ is clear from the context). Given three subsets $V_{1}, V_{2}, V_{3}$ of $V(H)$, we say that an edge $\left\{v_{1}, v_{2}, v_{3}\right\} \in E(H)$ is a type of $V_{1} V_{2} V_{3}$ if $v_{i} \in V_{i}$ for $1 \leqslant i \leqslant 3$. Given a vertex $v \in V(H)$ and a subset $A \subseteq V(H)$, we define the link $L_{v}(A)=\{u w: u, w \in A$ and $\{u, v, w\} \in E(H)\}$. When $A$ and $B$ are two disjoint subsets of $V(H)$, we let $L_{v}(A, B)=\{u w: u \in A, w \in B$ and $\{u, v, w\} \in E(H)\}$.

We write $0<a_{1} \ll a_{2} \ll a_{3}$ if we can choose the constants $a_{1}, a_{2}, a_{3}$ from right to left. More precisely there are increasing functions $f$ and $g$ such that given $a_{3}$, whenever we choose some $a_{2} \leqslant f\left(a_{3}\right)$ and $a_{1} \leqslant g\left(a_{2}\right)$, all calculations needed in our proof are valid.

\section{Outline of the proof and preliminaries}

Let $n$ be sufficiently large and $\tau n<s \leqslant n / 3$. Suppose $H$ is a 3-graph of order $n$ without an isolated vertex and $\sigma_{2}(H)>2 s n-\varepsilon n^{2}$. Let $U=\left\{u \in V(H): \operatorname{deg}(u)>s n-\frac{\varepsilon}{2} n^{2}\right\}$ and $W=V \backslash U$. Then any two vertices of $W$ are not adjacent - otherwise $\sigma_{2}(H) \leqslant 2 s n-\varepsilon n^{2}$, a contradiction. If $|U| \leqslant 2 s-1$, then $H$ is a subgraph of $H_{n, s}^{2}$ and we are done. We thus assume that $|U| \geqslant 2 s$.

Throughout the proof we use small constants

$$
0<\varepsilon \ll \varepsilon^{\prime} \ll \varepsilon^{\prime \prime} \ll \eta_{1} \ll \eta_{2} \ll \gamma \ll \gamma^{\prime} \ll \tau \ll 1 .
$$

We first prove the following lemma, which is an extension of [26, Lemma 4].

Lemma 7. Given $0<\varepsilon \ll \tau \ll 1$, let $n$ be sufficiently large and $\tau n<s \leqslant n / 3$. Suppose $H$ is a 3-graph of order $n$ without an isolated vertex and $\sigma_{2}(H)>2 s n-\varepsilon n^{2}$. Let 
$U=\left\{u \in V(H): \operatorname{deg}(u)>s n-\varepsilon n^{2} / 2\right\}$ and $W=V \backslash U$. If $2 s \leqslant|U| \leqslant 3 s$, then $H$ contains a matching of size $3 s-|U|$, each of which contains exactly one vertex of $W$.

Definition 8. We call a matching $M$ optimal if (i) $M$ contains a submatching $M_{1}=\{e \in$ $M: e \cap W \neq \emptyset\}$ of size at least $3 s-|U|$; (ii) subject to (i), $|M|$ is as large as possible; (iii) subject to (i) and (ii), $\left|M_{1}\right|$ is as large as possible.

Lemma 7 shows that $H$ contains an optimal matching $M$. We separate the cases when $\left|M_{1}\right|=3 s-|U|$ and when $\left|M_{1}\right|>3 s-|U|$. When $\left|M_{1}\right|=3 s-|U|$, we first consider the case when $s \leqslant n / 3-\eta_{1} n$. If no vertex of $U_{3}:=U \backslash V(M)$ is adjacent to any vertex of $W_{2}:=W \backslash V(M)$, then the assumption $\left|M_{1}\right|=3 s-|U|$ forces $\sum_{i=1}^{3} \operatorname{deg}\left(u_{i}\right)$ to be smaller than $3 s n-\frac{3}{2} \varepsilon n^{2}$ for any three vertices $u_{1}, u_{2}, u_{3} \in U_{3}$. If some vertex $u_{1} \in U_{3}$ is adjacent to $v_{1} \in W_{2}$, then the fact $v_{1} \in W$ reduces $\sum_{i=1}^{2} \operatorname{deg}\left(u_{i}\right)+\operatorname{deg}\left(v_{1}\right)$ to a number less than $3 s n-\frac{3}{2} \varepsilon n^{2}$ (where $u_{2}$ is another vertex of $U_{3}$ ). When $s>n / 3-\eta_{1} n$, we consider $H^{\prime}=H\left[V \backslash W_{2}\right]$. Since $\left|W_{2}\right|=n-3 s$ is very small, we deduce that $\sigma_{2}\left(H^{\prime}\right)$ is greater than $2 s n-\eta_{2} n^{2}$. This allows us to apply the following theorem from [26] to obtain a perfect matching of $H^{\prime}$, which is also a matching of size $s$ of $H$.

Theorem 9. [26] There exist $\eta_{2}>0$ and $n_{0} \in \mathbb{N}$ such that the following holds for all integers $n \geqslant n_{0}$ that are divisible by 3. Suppose that $H$ is a 3-graph of order $n$ without an isolated vertex and $\sigma_{2}(H)>2 n^{2} / 3-\eta_{2} n^{2}$, then either $H$ is a subgraph of $H_{n, n / 3}^{2}$ or $H$ contains a perfect matching.

Now consider the case when $\left|M_{1}\right|>3 s-|U|$. Let $W^{\prime}:=\{v \in W: \operatorname{deg}(v) \leqslant$ $\left.s n-s^{2} / 2+\gamma^{\prime} n^{2}\right\}$. If $\left|W^{\prime}\right|$ is very small, then we can find a matching of size $s$ in $H\left[V \backslash W^{\prime}\right]$ by Theorem 1 . When $\left|W^{\prime}\right|$ is not small, we consider $u_{1}, u_{2}, u_{3} \in U_{3}$. If one of $u_{1}, u_{2}$, $u_{3}$ is adjacent to one vertex from $W^{\prime}$, then $\sum_{i=1}^{3} \operatorname{deg}\left(u_{i}\right)$ becomes much larger than $3 s n$; otherwise we show that $\sum_{i=1}^{3} \operatorname{deg}\left(u_{i}\right)<3 s n-\frac{3}{2} \varepsilon n^{2}$ by proceeding with the cases when $\left|W^{\prime} \cap W_{1}\right|>\gamma n / 2$ and when $\left|W^{\prime} \cap W_{2}\right|>\gamma n / 2$ separately.

In the proof we need several (simple) extremal results on (hyper)graphs. Lemma 10 is Observation 1.8 of Aharoni and Howard [1]. Lemmas 11 and 12 are from [26]. A $k$-graph $H$ is called $k$-partite if $V(H)$ can be partitioned into $V_{1}, \ldots, V_{k}$, such that each edge of $H$ meets every $V_{i}$ in precisely one vertex. If all parts are of the same size $n$, we call $H$ $n$-balanced.

Lemma 10. [1] Let $F$ be the edge set of an $n$-balanced $k$-partite $k$-graph. If $F$ does not contain s disjoint edges, then $|F| \leqslant(s-1) n^{k-1}$.

Lemma 11. [26] Let $G_{1}, G_{2}, G_{3}$ be three graphs on the same set $V$ of $n \geqslant 4$ vertices such that every edge of $G_{1}$ intersects every edge of $G_{i}$ for both $i=2,3$. Then $\sum_{i=1}^{3} \sum_{v \in A} \operatorname{deg}_{G_{i}}(v) \leqslant 6(n-1)$ for any set $A \subset V$ of size 3 .

Lemma 12. [26] Let $G_{1}, G_{2}, G_{3}$ be three graphs on the same set $V$ of $n \geqslant 5$ vertices such that for any $i \neq j$, every edge of $G_{i}$ intersects every edge of $G_{j}$. Then $\sum_{i=1}^{3} \sum_{v \in A} \operatorname{deg}_{G_{i}}(v) \leqslant 3(n+1)$ for any set $A \subset V$ of size 3 . 
Following the same proof of Lemmas 11 and 12 from [26], we obtain another lemma and omit its proof.

Lemma 13. Let $G_{1}, \ldots, G_{k}$ be $k$ graphs on the same set $V$ of $n \geqslant 4$ vertices such that for any $1 \leqslant i<j \leqslant k$, every edge of $G_{i}$ intersects every edge of $G_{j}$. Then $\sum_{i=1}^{k} \sum_{v \in A} \operatorname{deg}_{G_{i}}(v) \leqslant k n$ for any set $A \subset V$ of size 2 .

The following lemma needs slightly more work so we include a proof.

Lemma 14. Given two disjoint vertex sets $A=\left\{u_{1}, u_{2}, \ldots, u_{a}\right\}$ and $B=\left\{v_{1}, v_{2}, \ldots, v_{b}\right\}$ with $a \geqslant 3$ and $b \geqslant 1$. Let $G_{i}, i=1,2,3$, be graphs on $A \cup B$ such that every vertex of $B$ is an isolated vertex in $G_{1}$, and every edge of $G_{i}(i=2,3)$ contains at least one vertex of $A$. If there are no two disjoint edges (i) one from $G_{1}$ and the other from $G_{2}$ or $G_{3}$; or (ii) one from $G_{2}$ and the other from $G_{3}$, and at least one of them contains a vertex from $B$, then

$$
\sum_{i=1}^{3}\left(\sum_{j=1}^{2} \operatorname{deg}_{G_{i}}\left(u_{j}\right)+\operatorname{deg}_{G_{i}}\left(v_{1}\right)\right) \leqslant \max \{4 a+7,3 a+2 b+5\} .
$$

Proof. For convenience, let $s_{i}=\sum_{j=1}^{2} \operatorname{deg}_{G_{i}}\left(u_{j}\right)+\operatorname{deg}_{G_{i}}\left(v_{1}\right)$ for $i=1,2,3$ and $y=$ $s_{1}+s_{2}+s_{3}$. Below we show that $y \leqslant \max \{4 a+7,3 a+2 b+5\}$.

We first observe that if $\operatorname{deg}_{G_{i}}\left(v_{1}\right) \geqslant 3$ for some $i \in\{2,3\}$, then $E\left(G_{1}\right)=\emptyset$ and $G_{i^{\prime}}$ is a star centered at $v_{1}$, where $i^{\prime}=5-i$. Indeed, if $G_{1}$ or $G_{i^{\prime}}$ contains an edge $e$ not incident to $v_{1}$, then $e$ is disjoint from some edge of $G_{i}$ that is incident to $v_{1}$ - this contradicts our assumption. The observation implies that if $\operatorname{deg}_{G_{i}}\left(v_{1}\right) \geqslant 3$ for both $i=2,3$, then $E\left(G_{1}\right)=\emptyset$ and both $G_{2}$ and $G_{3}$ are stars centered at $v_{1}$. In this case, $s_{i} \leqslant a+2$ for $i=2,3$ and thus $y \leqslant 2(a+2)$. If $\operatorname{deg}_{G_{2}}\left(v_{1}\right) \geqslant 3$ and $\operatorname{deg}_{G_{3}}\left(v_{1}\right) \leqslant 2$, then $E\left(G_{1}\right)=\emptyset$ and $G_{3}$ consists of at most two edges incident to $v_{1}$. In this case, $s_{1} \leqslant 2(a+b-1)+a, s_{2} \leqslant 4$ and thus $y \leqslant 3 a+2 b+2$. The case when $\operatorname{deg}_{G_{2}}\left(v_{1}\right) \leqslant 2$ and $\operatorname{deg}_{G_{3}}\left(v_{1}\right) \geqslant 3$ is analogous. We thus assume that

$$
\operatorname{deg}_{G_{i}}\left(v_{1}\right) \leqslant 2 \text { for } i=2,3
$$

for the rest of the proof.

Next, we observe that if $\left|N_{G_{i}}\left(u_{j}\right) \cap B\right| \geqslant 2$ for some $i \in\{2,3\}$ and some $j \in\{1,2\}$, then $G_{i^{\prime}}$ is a star centered at $u_{j}$ for $i^{\prime} \in\{1,2,3\} \backslash\{i\}$. This is again due to our assumption on $G_{1}, G_{2}$ and $G_{3}$. The observation implies that if $\left|N_{G_{i}}\left(u_{j}\right) \cap B\right| \geqslant 2$ for both $j=1,2$, then $E\left(G_{i^{\prime}}\right) \subseteq\left\{u_{1} u_{2}\right\}$ and consequently, $s_{i^{\prime}} \leqslant 2$ for $i^{\prime} \in\{1,2,3\} \backslash\{i\}$. By (2), we have $s_{i} \leqslant 2(a+b-1)+2$. Therefore, $y \leqslant 2(a+b-1)+2+4=2 a+2 b+4$. The observation also implies that if $\left|N_{G_{i}}\left(u_{j}\right) \cap B\right| \geqslant 2$ for both $i=2,3$, then $G_{1}, G_{2}, G_{3}$ are all stars centered at $u_{j}$. In this case, $s_{1} \leqslant a$ and $s_{i} \leqslant a+b+1$ for $i=2,3$, which implies that $y \leqslant a+2(a+b+1)=3 a+2 b+2$. We now consider the case when $\left|N_{G_{2}}\left(u_{1}\right) \cap B\right| \geqslant 2,\left|N_{G_{2}}\left(u_{2}\right) \cap B\right| \leqslant 1$, and $\left|N_{G_{3}}\left(u_{1}\right) \cap B\right| \leqslant 1$. Thus $G_{3}$ is a star (centered at $u_{1}$ ) of size at most $a$, which yields $s_{3} \leqslant a+2$. Now suppose $N_{G_{2}}\left(u_{2}\right) \cap B \subseteq\left\{v_{p}\right\}$ for some $p$. Let $A^{\prime}:=A \cup\left\{v_{p}\right\}$ (note that $\left|A^{\prime}\right|=a+1 \geqslant 4$ ). Since every edge of $G_{1}$ 
intersects every edge of $G_{2}$, we can apply Lemma 13 to $G_{1}\left[A^{\prime}\right]$ and $G_{2}\left[A^{\prime}\right]$ and obtain that $\sum_{i=1}^{2} \sum_{j=1}^{2} \operatorname{deg}_{G_{i}\left[A^{\prime}\right]}\left(u_{j}\right) \leqslant 2 a+2$. Since $\left|N_{G_{2}}\left(u_{1}\right) \cap\left(B \backslash\left\{v_{p}\right\}\right)\right| \leqslant b-1$ and $\operatorname{deg}_{G_{2}}\left(v_{1}\right) \leqslant 2$, it follows that $s_{1}+s_{2} \leqslant 2 a+2+b-1+2=2 a+b+3$ and $y \leqslant 2 a+b+3+a+2=3 a+b+5$.

We thus assume that $\left|N_{G_{i}}\left(u_{j}\right) \cap B\right| \leqslant 1$ for $i=2,3$ and $j=1,2$. Suppose $N_{G_{2}}\left(u_{2}\right) \cap B \subseteq$ $\left\{v_{p}\right\}$ for some $p$ and let $A^{\prime}:=A \cup\left\{v_{p}\right\}$. We apply Lemma 13 to $G_{1}\left[A^{\prime}\right]$ and $G_{2}\left[A^{\prime}\right]$ and obtain that $\sum_{i=1}^{2} \sum_{j=1}^{2} \operatorname{deg}_{G_{i}\left[A^{\prime}\right]}\left(u_{j}\right) \leqslant 2 a+2$. Since $\left|N_{G_{2}}\left(u_{1}\right) \cap B\right| \leqslant 1$ and $\operatorname{deg}_{G_{2}}\left(v_{1}\right) \leqslant 2$, it follows that $s_{1}+s_{2} \leqslant 2 a+2+1+2$. On the other hand, we have $s_{3} \leqslant 2 a+2$ because $\operatorname{deg}_{G_{3}}\left(u_{j}\right) \leqslant a$ for $j=1,2$ and $\operatorname{deg}_{G_{3}}\left(v_{1}\right) \leqslant 2$. Thus $y \leqslant 2 a+5+2 a+2=4 a+7$.

\section{Proof of Lemma 7}

The proof is similar to that of [26, Lemma 4]. Let $M$ be a largest matching of $H$ such that each edge of $M$ contains (exactly) one vertex of $W$. To the contrary, assume $|M| \leqslant$ $3 s-|U|-1$. Let $U_{1}=V(M) \cap U, U_{2}=U \backslash U_{1}, W_{1}=V(M) \cap W$ and $W_{2}=W \backslash W_{1}$. Since $|U| \geqslant 2 s$, we have $\left|U_{2}\right|=|U|-2|M| \geqslant 2$. Since $\left|W_{2}\right|=|W|-|M|$ and $|W| \geqslant 3 s-|U|$, it follows that $W_{2} \neq \emptyset$.

Below is a sketch of the proof. We first assume $|U|<2 s+\varepsilon^{\prime} n$. In this case every vertex in $U$ is adjacent to some vertex in $W$. If $|M|$ is not close to $s$, then we easily obtain a contradiction because $U_{2}$ is not small. When $|M|$ is close to $s$, we consider three vertices $u_{1} \neq u_{2} \in U_{2}$ and $v_{0} \in W_{2}$, and derive a contradiction on $\operatorname{deg}\left(u_{1}\right)+\operatorname{deg}\left(u_{2}\right)+\operatorname{deg}\left(v_{0}\right)$. Next we assume $|U| \geqslant 2 s+\varepsilon^{\prime} n$. In this case $U_{2}$ is not small. If no vertex of $W_{2}$ is adjacent to any vertex of $U_{2}$, then consider two adjacent vertices $v_{0} \in W_{2}$ and $u_{0} \in U_{1}$. We have $\operatorname{deg}\left(v_{0}\right) \leqslant\left(\begin{array}{c}2|M| \\ 2\end{array}\right)$, which eventually yields that $\operatorname{deg}\left(v_{0}\right)+\operatorname{deg}\left(u_{0}\right)<2 s n-\varepsilon n^{2}$. Now assume $v_{0} \in W_{2}$ is adjacent to some vertex $u_{0} \in U_{2}$. In this case we define $M^{\prime}$ consisting of all $e \in M$ that contains a vertex $u^{\prime} \in U$ such that $\left|N\left(v_{0}, u^{\prime}\right) \cap U_{2}\right| \geqslant 3$. We show that if $\left|M^{\prime}\right|$ is small, then $\operatorname{deg}\left(v_{0}\right)$ is small; otherwise $\operatorname{deg}\left(u_{0}\right)$ is small. In either case we derive that $\operatorname{deg}\left(v_{0}\right)+\operatorname{deg}\left(u_{0}\right)<2 s n-\varepsilon n^{2}$.

We now give the details of the proof.

Case 1. $2 s \leqslant|U|<2 s+\varepsilon^{\prime} n$.

In this case we have the following two claims.

Claim 15. $|M| \geqslant s-\varepsilon^{\prime \prime} n$.

Proof. To the contrary, assume that $|M|<s-\varepsilon^{\prime \prime} n$. Fix $v_{0} \in W_{2}$. Then $\operatorname{deg}\left(v_{0}\right) \leqslant$ $\left(\begin{array}{c}|U| \\ 2\end{array}\right)-\left(\begin{array}{c}\left|U_{2}\right| \\ 2\end{array}\right)$ because there is no edge of type $U_{2} U_{2} W_{2}$. Since $v_{0}$ is not an isolated vertex, $v_{0}$ is adjacent to some vertex $u \in U$. Trivially $\operatorname{deg}(u) \leqslant\left(\begin{array}{c}|U|-1 \\ 2\end{array}\right)+(|U|-1)|W|$. Thus

$$
\begin{aligned}
\operatorname{deg}\left(v_{0}\right)+\operatorname{deg}(u) & \leqslant\left(\begin{array}{c}
|U|-1 \\
2
\end{array}\right)+(|U|-1)|W|+\left(\begin{array}{c}
|U| \\
2
\end{array}\right)-\left(\begin{array}{c}
\left|U_{2}\right| \\
2
\end{array}\right) \\
& =(n-1)(|U|-1)-\left(\begin{array}{c}
\left|U_{2}\right| \\
2
\end{array}\right) .
\end{aligned}
$$

Since $|U| \geqslant 2 s$ and $|M|<s-\varepsilon^{\prime \prime} n$, it follows that $\left|U_{2}\right|=|U|-2|M|>2 \varepsilon^{\prime \prime} n$. As a result,

$$
\operatorname{deg}(u)+\operatorname{deg}\left(v_{0}\right) \leqslant(n-1)\left(2 s+\varepsilon^{\prime} n-1\right)-\left(\begin{array}{c}
2 \varepsilon^{\prime \prime} n \\
2
\end{array}\right),
$$


which contradicts the condition that $\operatorname{deg}(u)+\operatorname{deg}\left(v_{0}\right)>2 s n-\varepsilon n^{2}$ because $\varepsilon \ll \varepsilon^{\prime} \ll$ $\varepsilon^{\prime \prime}$.

Claim 16. Every vertex in $U$ is adjacent to one vertex in $W$.

Proof. To the contrary, assume that $u \in U$ is not adjacent to any vertex in $W$. Then

$$
\operatorname{deg}(u) \leqslant\left(\begin{array}{c}
|U|-1 \\
2
\end{array}\right)<\left(\begin{array}{c}
2 s+\varepsilon^{\prime} n \\
2
\end{array}\right)
$$

which contradicts the condition that $\operatorname{deg}(u)>s n-\frac{1}{2} \varepsilon n^{2}$ because $\tau n<s \leqslant n / 3$ and $\varepsilon \ll \varepsilon^{\prime} \ll \tau$.

Fix $u_{1} \neq u_{2} \in U_{2}$ and $v_{0} \in W_{2}$. Trivially $\operatorname{deg}(w) \leqslant\left(\begin{array}{c}|U| \\ 2\end{array}\right)$ for any vertex $w \in W$ and $\operatorname{deg}(u) \leqslant\left(\begin{array}{c}|U|-1 \\ 2\end{array}\right)+|W|(|U|-1)$ for any vertex $u \in U$. Furthermore, for any two distinct edges $e_{1}, e_{2} \in M$, we observe that at least one triple of type $U U W$ with one vertex in $e_{1}$, one vertex in $e_{2}$ and one vertex in $\left\{u_{1}, u_{2}, v_{0}\right\}$ is not an edge by the choice of $M$. By Claim $15,|M| \geqslant s-\varepsilon^{\prime \prime} n$. Thus,

$$
\operatorname{deg}\left(u_{1}\right)+\operatorname{deg}\left(u_{2}\right)+\operatorname{deg}\left(v_{0}\right) \leqslant 2\left(\left(\begin{array}{c}
|U|-1 \\
2
\end{array}\right)+|W|(|U|-1)\right)+\left(\begin{array}{c}
|U| \\
2
\end{array}\right)-\left(\begin{array}{c}
s-\varepsilon^{\prime \prime} n \\
2
\end{array}\right) .
$$

On the other hand, Claim 16 implies that $u_{i}$ is adjacent to some vertex in $W$ for $i=1,2$. We know that $v_{0}$ is adjacent to some vertex in $U$. Therefore, $\operatorname{deg}\left(u_{i}\right)>\left(2 s n-\varepsilon n^{2}\right)-\left(\begin{array}{c}|U| \\ 2\end{array}\right)$ for $i=1,2$, and $\operatorname{deg}\left(v_{0}\right)>\left(2 s n-\varepsilon n^{2}\right)-\left(\left(\begin{array}{c}|U|-1 \\ 2\end{array}\right)+|W|(|U|-1)\right)$. It follows that

$$
\operatorname{deg}\left(u_{1}\right)+\operatorname{deg}\left(u_{2}\right)+\operatorname{deg}\left(v_{0}\right)>3\left(2 s n-\varepsilon n^{2}\right)-2\left(\begin{array}{c}
|U| \\
2
\end{array}\right)-\left(\begin{array}{c}
|U|-1 \\
2
\end{array}\right)-|W|(|U|-1) .
$$

The upper and lower bounds for $\operatorname{deg}\left(u_{1}\right)+\operatorname{deg}\left(u_{2}\right)+\operatorname{deg}\left(v_{0}\right)$ together imply that

$$
\begin{aligned}
3\left(\left(\begin{array}{c}
|U|-1 \\
2
\end{array}\right)+|W|(|U|-1)+\left(\begin{array}{c}
|U| \\
2
\end{array}\right)\right)-\left(\begin{array}{c}
s-\varepsilon^{\prime \prime} n \\
2
\end{array}\right) & >3\left(2 s n-\varepsilon n^{2}\right), \\
\text { or } \quad(|U|-1)(n-1)-\frac{1}{3}\left(\begin{array}{c}
s-\varepsilon^{\prime \prime} n \\
2
\end{array}\right) & >2 s n-\varepsilon n^{2},
\end{aligned}
$$

which is impossible because $|U|<2 s+\varepsilon^{\prime} n, \tau n<s \leqslant n / 3$, and $\varepsilon \ll \varepsilon^{\prime} \ll \varepsilon^{\prime \prime} \ll \tau$.

Case 2. $2 s+\varepsilon^{\prime} n \leqslant|U| \leqslant 3 s$.

We consider the following two subcases.

Subcase 2.1. No vertex in $U_{2}$ is adjacent to any vertex in $W_{2}$.

Fix $v_{0} \in W_{2}$. Then $\operatorname{deg}\left(v_{0}\right) \leqslant\left(\begin{array}{c}\left|U_{1}\right| \\ 2\end{array}\right)=\left(\begin{array}{c}2|M| \\ 2\end{array}\right)$. Since $v_{0}$ is not an isolated vertex, $v_{0}$ is adjacent to some vertex $u_{0} \in U_{1}$. We know that $\operatorname{deg}\left(u_{0}\right) \leqslant\left(\begin{array}{c}|U|-1 \\ 2\end{array}\right)+(|U|-1)|W|-\left|U_{2}\right|\left|W_{2}\right|$ 
because no vertex in $U_{2}$ is adjacent to any vertex in $W_{2}$. Since $|W|=n-|U|,\left|U_{2}\right|=$ $|U|-2|M|$ and $\left|W_{2}\right|=n-|U|-|M|$, we derive that

$$
\begin{aligned}
\sigma_{2}(H) & \leqslant \operatorname{deg}\left(v_{0}\right)+\operatorname{deg}\left(u_{0}\right) \\
& \leqslant\left(\begin{array}{c}
2|M| \\
2
\end{array}\right)+\left(\begin{array}{c}
|U|-1 \\
2
\end{array}\right)+(|U|-1)(n-|U|)-(|U|-2|M|)(n-|U|-|M|) \\
& \leqslant(2 n-|U|)|M|+\frac{|U|^{2}}{2} .
\end{aligned}
$$

Since $|M|<3 s-|U|$, it follows that

$$
\sigma_{2}(H)<(2 n-|U|)(3 s-|U|)+\frac{|U|^{2}}{2}=6 s n-(3 s+2 n)|U|+\frac{3}{2}|U|^{2} .
$$

Note that the quadratic function $\frac{3}{2} x^{2}-(3 s+2 n) x$ is minimized at $x=s+\frac{2}{3} n$. Since $2 s+\varepsilon^{\prime} n \leqslant|U| \leqslant 3 s \leqslant s+\frac{2}{3} n$, we derive that

$$
\begin{aligned}
\sigma_{2}(H) & \leqslant 6 s n-(3 s+2 n)\left(2 s+\varepsilon^{\prime} n\right)+\frac{3}{2}\left(2 s+\varepsilon^{\prime} n\right)^{2} \\
& =2 s n-2 \varepsilon^{\prime} n^{2}+3 s \varepsilon^{\prime} n+\frac{3}{2} \varepsilon^{\prime 2} n^{2} \leqslant 2 s n-\varepsilon^{\prime} n^{2}+\frac{3}{2} \varepsilon^{\prime 2} n^{2}
\end{aligned}
$$

because $s \leqslant n / 3$. Since $\varepsilon \ll \varepsilon^{\prime}$, this contradicts the assumption that $\sigma_{2}(H)>2 s n-\varepsilon n$.

Subcase 2.2. Two vertices $u_{0} \in U_{2}$ and $v_{0} \in W_{2}$ are adjacent.

Let $M^{\prime}=\left\{e \in M: \exists u^{\prime} \in e,\left|N\left(v_{0}, u^{\prime}\right) \cap U_{2}\right| \geqslant 3\right\}$. Assume $\left\{u_{1}, u_{2}, v_{1}\right\} \in M^{\prime}$ such that $u_{1}, u_{2} \in U_{1}, v_{1} \in W_{1}$ and $\left|N\left(v_{0}, u_{1}\right) \cap U_{2}\right| \geqslant 3$. We claim that

$$
N\left(u_{0}, v_{1}\right) \cap U_{2}=\emptyset .
$$

Indeed, if $\left\{u_{0}, v_{1}, u_{3}\right\} \in E(H)$ for some $u_{3} \in U_{2}$, then we can find $u_{4} \in U_{2} \backslash\left\{u_{0}, u_{3}\right\}$ such that $\left\{v_{0}, u_{1}, u_{4}\right\} \in E(H)$. Replacing $\left\{u_{1}, u_{2}, v_{1}\right\}$ by $\left\{u_{0}, v_{1}, u_{3}\right\}$ and $\left\{v_{0}, u_{1}, u_{4}\right\}$ gives a larger matching than $M$, a contradiction.

By the definition of $M^{\prime}$, we have

$$
\operatorname{deg}\left(v_{0}\right) \leqslant\left(\begin{array}{c}
\left|U_{1}\right| \\
2
\end{array}\right)+2\left|M^{\prime}\right|\left|U_{2}\right|+2\left(\left|U_{1}\right|-2\left|M^{\prime}\right|\right)=\left(\begin{array}{c}
\left|U_{1}\right| \\
2
\end{array}\right)+2\left|U_{1}\right|+\left|M^{\prime}\right|\left(2\left|U_{2}\right|-4\right) .
$$

By (3), we have

$$
\operatorname{deg}\left(u_{0}\right) \leqslant\left(\begin{array}{c}
|U|-1 \\
2
\end{array}\right)+\left|U_{1}\right||W|+\left(\left|U_{2}\right|-1\right)\left(\left|W_{1}\right|-\left|M^{\prime}\right|\right)
$$

and consequently

$\operatorname{deg}\left(v_{0}\right)+\operatorname{deg}\left(u_{0}\right) \leqslant\left(\begin{array}{c}\left|U_{1}\right| \\ 2\end{array}\right)+\left(\begin{array}{c}|U|-1 \\ 2\end{array}\right)+\left|U_{1}\right|(|W|+2)+\left(\left|U_{2}\right|-1\right)\left|W_{1}\right|+\left|M^{\prime}\right|\left(\left|U_{2}\right|-3\right)$. 
Since $\left|M^{\prime}\right| \leqslant|M|=\left|W_{1}\right|=\frac{\left|U_{1}\right|}{2}$, it follows that

$$
\begin{aligned}
\operatorname{deg}\left(v_{0}\right)+\operatorname{deg}\left(u_{0}\right) & \leqslant\left(\begin{array}{c}
\left|U_{1}\right| \\
2
\end{array}\right)+\left(\begin{array}{c}
|U|-1 \\
2
\end{array}\right)+\left|U_{1}\right|(|W|+2)+\left(\left|U_{2}\right|-2\right)\left|U_{1}\right| \\
& =\left(\begin{array}{c}
|U| \\
2
\end{array}\right)-\left(\begin{array}{c}
\left|U_{2}\right| \\
2
\end{array}\right)+\left(\begin{array}{c}
|U|-1 \\
2
\end{array}\right)+\left|U_{1}\right||W| \\
& =(|U|-1)^{2}-\left(\begin{array}{c}
\left|U_{2}\right| \\
2
\end{array}\right)+2|M|(n-|U|) .
\end{aligned}
$$

Since $|M| \leqslant 3 s-|U|$ and $\left|U_{2}\right|=|U|-2|M| \geqslant 3|U|-6 s$, we have

$$
\begin{aligned}
\operatorname{deg}\left(v_{0}\right)+\operatorname{deg}\left(u_{0}\right) & \leqslant(|U|-1)^{2}-\left(\begin{array}{c}
3|U|-6 s \\
2
\end{array}\right)+2(3 s-|U|)(n-|U|) \\
& =-\frac{3}{2}|U|^{2}+\left(12 s-2 n-\frac{1}{2}\right)|U|+6 s n-18 s^{2}-3 s+1 \\
& \leqslant-\frac{3}{2}|U|^{2}+(12 s-2 n)|U|+6 s n-18 s^{2} .
\end{aligned}
$$

Note that the quadratic function $-\frac{3}{2} x^{2}+(12 s-2 n) x$ is maximized at $x=4 s-\frac{2}{3} n$. Since $3 s \geqslant|U| \geqslant 2 s+\varepsilon^{\prime} n \geqslant 4 s-\frac{2}{3} n$, we have

$$
\begin{aligned}
\sigma_{2}(H) \leqslant \operatorname{deg}\left(v_{0}\right)+\operatorname{deg}\left(u_{0}\right) & \leqslant-\frac{3}{2}\left(2 s+\varepsilon^{\prime} n\right)^{2}+(12 s-2 n)\left(2 s+\varepsilon^{\prime} n\right)+6 s n-18 s^{2} \\
& =2 s n-2 \varepsilon^{\prime} n^{2}+6 \varepsilon^{\prime} s n-\frac{3}{2} \varepsilon^{\prime 2} n^{2} \leqslant 2 s n-\frac{3}{2} \varepsilon^{\prime 2} n^{2}
\end{aligned}
$$

because $s \leqslant n / 3$. Since $\varepsilon \ll \varepsilon^{\prime}$, this contradicts the assumption that $\sigma_{2}(H)>2 s n-\varepsilon n$.

\section{Proof of Theorem 6}

Suppose $H$ is a 3 -graph of order $n$ without an isolated vertex and $\sigma_{2}(H)>2 s n-\varepsilon n^{2}$. Let $U=\left\{u \in V(H): \operatorname{deg}(u)>s n-\varepsilon n^{2} / 2\right\}$ and $W=V \backslash U$. We know that no two vertices in $W$ are adjacent and $|U| \geqslant 2 s$. Let $M$ be an optimal matching as in Definition 8 . By Lemma 7, such $M$ exists. Let $M_{2}=M \backslash M_{1}, U_{1}=V\left(M_{1}\right) \cap U, U_{2}=V\left(M_{2}\right)$, $U_{3}=U \backslash V(M), W_{1}=V\left(M_{1}\right) \cap W$ and $W_{2}=W \backslash W_{1}$. Since $M$ is optimal, no edge of $H$ is of type $W_{2} U_{3} U_{3}$ or $W_{2} U_{2} U_{3}$. In addition, for any $e \in M_{1}$, there are no two disjoint edges $e_{1}, e_{2} \in e \cup W_{2} \cup U_{3}$ such that $\left(e_{1} \cup e_{2}\right) \cap W_{2} \neq \emptyset$.

Suppose to the contrary, that $|M| \leqslant s-1$. We know that $\left|U_{3}\right|=|U|+\left|M_{1}\right|-3|M| \geqslant$ $3+\left|M_{1}\right|-(3 s-|U|) \geqslant 3$. Let $u_{1}, u_{2}, u_{3} \in U_{3}$. Since $u_{i} \in U$ for $i=1,2,3$, we have

$$
\sum_{i=1}^{3} \operatorname{deg}\left(u_{i}\right)>3 s n-\frac{3}{2} \varepsilon n^{2} .
$$

On the other hand, if $u_{1}$ is adjacent to some $v_{1} \in W_{2}$, then

$$
\sum_{i=1}^{2} \operatorname{deg}\left(u_{i}\right)+\operatorname{deg}\left(v_{1}\right) \geqslant \sigma_{2}(H)+\operatorname{deg}\left(u_{2}\right)>3 s n-\frac{3}{2} \varepsilon n^{2} .
$$


Claim 17. For any two distinct edges $e_{1}$, e $e_{2}$ from $M$, we have $\sum_{i=1}^{3}\left|L_{u_{i}}\left(e_{1}, e_{2}\right)\right| \leqslant 18$ and $\sum_{i=1}^{2}\left|L_{u_{i}}\left(e_{1}, e_{2}\right)\right|+\left|L_{v_{1}}\left(e_{1}, e_{2}\right)\right| \leqslant 18$.

Proof. Let $H_{1}$ be the 3-partite subgraph of $H$ induced on three parts $\left\{u_{1}, u_{2}, u_{3}\right\}, e_{1}$, and $e_{2}$. We observe that $H_{1}$ does not contain a perfect matching by the choice of $M$. By Lemma 10, we have $\left|E\left(H_{1}\right)\right|=\sum_{i=1}^{3}\left|L_{u_{i}}\left(e_{1}, e_{2}\right)\right| \leqslant 18$. The same argument shows that $\sum_{i=1}^{2}\left|L_{u_{i}}\left(e_{1}, e_{2}\right)\right|+\left|L_{v_{1}}\left(e_{1}, e_{2}\right)\right| \leqslant 18$.

We proceed in two cases.

Case 1. $\left|M_{1}\right|=3 s-|U|$.

In this case, we have $\left|M_{2}\right|=|M|+|U|-3 s,\left|U_{3}\right|=3 s-3|M|$ and $\left|W_{2}\right|=n-3 s$.

Claim 18. For any $e \in M_{1}$, we have

(i) $\sum_{i=1}^{2}\left|L_{u_{i}}\left(e, U_{3} \cup W_{2}\right)\right|+\left|L_{v_{1}}\left(e, U_{3} \cup W_{2}\right)\right| \leqslant \max \left\{4\left|U_{3}\right|+7,3\left|U_{3}\right|+2\left|W_{2}\right|+5\right\}$, where $v_{1} \in W_{2}$

(ii) $\sum_{i=1}^{3}\left|L_{u_{i}}\left(e, U_{3}\right)\right| \leqslant 6\left|U_{3}\right|$.

Proof. Assume $e=\left\{u_{1}^{\prime}, u_{2}^{\prime}, u_{3}^{\prime}\right\} \in M_{1}$ with $u_{1}^{\prime} \in W_{1}$ and $u_{2}^{\prime}, u_{3}^{\prime} \in U_{1}$.

(i) Let $A=U_{3}, B=W_{2}$, and $E\left(G_{i}\right)=L_{u_{i}^{\prime}}\left(U_{3} \cup W_{2}\right)$ for $i=1,2,3$. By the choice of $M$, there are not two disjoint edges, one from $G_{1}$ and the other from $G_{2}$ or $G_{3}$; or one from $G_{2}$ and the other from $G_{3}$, and at least one of them contains one vertex from $B$. Furthermore, it is easy to see that

$$
\sum_{i=1}^{2}\left|L_{u_{i}}\left(e, U_{3} \cup W_{2}\right)\right|+\left|L_{v_{1}}\left(e, U_{3} \cup W_{2}\right)\right|=\sum_{i=1}^{3}\left(\sum_{j=1}^{2} \operatorname{deg}_{G_{i}}\left(u_{j}\right)+\operatorname{deg}_{G_{i}}\left(v_{1}\right)\right) .
$$

The desired inequality thus follows from Lemma 14 .

(ii) For $i=1,2,3$, let $G_{i}$ be the graph obtained from $L_{u_{i}^{\prime}}\left(U_{3}\right)$ after adding an isolated vertex $u^{*}$. Then $\left|V\left(G_{i}\right)\right|=\left|U_{3}\right|+1 \geqslant 4$. By the choice of $M$, every edge of $G_{1}$ intersects every edge of $G_{2}$ and $G_{3}$. The desired inequality thus follows from Lemma 11.

Claim 19. For any $e \in M_{2}$, we have

(i) $\sum_{i=1}^{3}\left|L_{u_{i}}\left(e, U_{3}\right)\right| \leqslant 3\left(\left|U_{3}\right|+3\right)$;

(ii) $\sum_{i=1}^{2}\left|L_{u_{i}}\left(e, U_{3}\right)\right| \leqslant 3\left(\left|U_{3}\right|+1\right)$.

Proof. Assume $e=\left\{u_{1}^{\prime}, u_{2}^{\prime}, u_{3}^{\prime}\right\} \in M_{2}$ with $u_{1}^{\prime}, u_{2}^{\prime}, u_{3}^{\prime} \in U_{2}$.

(i) For $i=1,2,3$, let $G_{i}$ be the graph obtained from $L_{u_{i}^{\prime}}\left(U_{3}\right)$ after adding two isolated vertices $u^{\prime}$ and $u^{\prime \prime}$. Then $\left|V\left(G_{i}\right)\right|=\left|U_{3}\right|+2 \geqslant 5$. Since $M$ is optimal, the desired inequality follows from Lemma 12.

(ii) For $i=1,2,3$, let $G_{i}$ be the graph obtained from $L_{u_{i}^{\prime}}\left(U_{3}\right)$ after adding an isolated vertex $u^{*}$. Then $\left|V\left(G_{i}\right)\right|=\left|U_{3}\right|+1 \geqslant 4$. Since $M$ is optimal, the desired inequality follows from Lemma 13. 
Claim 20. $s>n / 3-\eta_{1} n$.

Proof. Suppose $s \leqslant n / 3-\eta_{1} n$. We first consider the case that $u_{1}, u_{2}, u_{3}$ are not adjacent to any vertex of $W_{2}$.

Following Claim 17, we have

$$
\sum_{i=1}^{3} \operatorname{deg}\left(u_{i}\right) \leqslant 18\left(\begin{array}{c}
|M| \\
2
\end{array}\right)+9|M|+\sum_{i=1}^{3}\left|L_{u_{i}}\left(V\left(M_{1}\right), U_{3}\right)\right|+\sum_{i=1}^{3}\left|L_{u_{i}}\left(V\left(M_{2}\right), U_{3}\right)\right| .
$$

Furthermore, by Claims 18 (ii) and 19 (i), we obtain that

$$
\begin{aligned}
\sum_{i=1}^{3} \operatorname{deg}\left(u_{i}\right) \leqslant & 18\left(\begin{array}{c}
|M| \\
2
\end{array}\right)+9|M|+6\left|M_{1}\right|\left|U_{3}\right|+3\left|M_{2}\right|\left(\left|U_{3}\right|+3\right) \\
= & 18\left(\begin{array}{c}
|M| \\
2
\end{array}\right)+9|M|+6(3 s-|U|)(3 s-3|M|) \\
& +3(|M|+|U|-3 s)(3 s-3|M|+3) \\
= & (9|U|-18 s+9)|M|+(3 s-|U|)(9 s-9) .
\end{aligned}
$$

Since $|M| \leqslant s-1$, it follows that

$$
\sum_{i=1}^{3} \operatorname{deg}\left(u_{i}\right) \leqslant(9|U|-18 s+9)(s-1)+(3 s-|U|)(9 s-9)=9 s^{2}-9 .
$$

Since $\tau n<s \leqslant n / 3-\eta_{1} n$ and $\eta_{1}<\tau$, we know that

$$
3 s^{2}-s n=s(3 s-n) \leqslant \max \left\{-\eta_{1} n\left(n-3 \eta_{1} n\right),-\tau n(n-3 \tau n)\right\}=-\eta_{1} n\left(n-3 \eta_{1} n\right) .
$$

Consequently, $\sum_{i=1}^{3} \operatorname{deg}\left(u_{i}\right)<9 s^{2} \leqslant 3 s n-3 \eta_{1} n\left(n-3 \eta_{1} n\right)$. Since $\varepsilon \ll \eta_{1}$, this contradicts (4).

Now we assume, without loss of generality, that $u_{1}$ is adjacent to $v_{1}$. The choice of $M$ implies that $L_{v}\left(e, U_{3}\right)=L_{u}\left(e, W_{2}\right)=\emptyset$ for any $v \in W_{2}, u \in U_{3}$ and $e \in M_{2}$. By Claim 17, we have

$$
\begin{aligned}
\sum_{i=1}^{2} \operatorname{deg}\left(u_{i}\right)+\operatorname{deg}\left(v_{1}\right) \leqslant & 18\left(\begin{array}{c}
|M| \\
2
\end{array}\right)+9|M|+\sum_{i=1}^{2}\left|L_{u_{i}}\left(V\left(M_{1}\right), U_{3} \cup W_{2}\right)\right| \\
& +\left|L_{v_{1}}\left(V\left(M_{1}\right), U_{3}\right)\right|+\sum_{i=1}^{2}\left|L_{u_{i}}\left(V\left(M_{2}\right), U_{3}\right)\right|
\end{aligned}
$$


We know that $4\left|U_{3}\right|+7 \geqslant 3\left|U_{3}\right|+2\left|W_{2}\right|+5$ if and only if $\left|U_{3}\right| \geqslant 2\left|W_{2}\right|-2$. If $\left|U_{3}\right| \geqslant 2\left|W_{2}\right|-2$, then by (8), Claim 18 (i) and Claim 19 (ii), we have

$$
\begin{aligned}
\sum_{i=1}^{2} \operatorname{deg}\left(u_{i}\right)+\operatorname{deg}\left(v_{1}\right) \leqslant & 18\left(\begin{array}{c}
|M| \\
2
\end{array}\right)+9|M|+\left|M_{1}\right|\left(4\left|U_{3}\right|+7\right)+3\left|M_{2}\right|\left(\left|U_{3}\right|+1\right) \\
= & 18\left(\begin{array}{c}
|M| \\
2
\end{array}\right)+9|M|+(3 s-|U|)(4(3 s-3|M|)+7) \\
& +3(|M|+|U|-3 s)(3 s-3|M|+1) \\
= & (3|U|+3)|M|-3 s|U|-4|U|+9 s^{2}+12 s .
\end{aligned}
$$

Since $|M| \leqslant s-1$ and $|U| \geqslant 2 s$, it follows that

$$
\begin{aligned}
\sum_{i=1}^{2} \operatorname{deg}\left(u_{i}\right)+\operatorname{deg}\left(v_{1}\right) & \leqslant(3|U|+3)(s-1)-3 s|U|-4|U|+9 s^{2}+12 s \\
& =-7|U|+9 s^{2}+15 s-3 \leqslant 9 s^{2}+s-3 .
\end{aligned}
$$

Following (7), we have $\sum_{i=1}^{2} \operatorname{deg}\left(u_{i}\right)+\operatorname{deg}\left(v_{1}\right)<3 s n-3 \eta_{1} n\left(n-3 \eta_{1} n\right)+n / 3-3$. Since $\varepsilon \ll \eta_{1}$ and $n$ is sufficiently large, this contradicts (5).

If $\left|U_{3}\right|<2\left|W_{2}\right|-2$, by (8), Claim 18 (i) and Claim 19 (ii), we have

$$
\begin{aligned}
\sum_{i=1}^{2} \operatorname{deg}\left(u_{i}\right)+\operatorname{deg}\left(v_{1}\right) & \leqslant 18\left(\begin{array}{c}
|M| \\
2
\end{array}\right)+9|M|+\left|M_{1}\right|\left(3\left|U_{3}\right|+2\left|W_{2}\right|+5\right)+3\left|M_{2}\right|\left(\left|U_{3}\right|+1\right) \\
& =(9 s+3)|M|+(-2 n+6 s-2)|U|+6 s n-18 s^{2}+6 s .
\end{aligned}
$$

Since $|M| \leqslant s-1$ and $|U| \geqslant 2 s$, it follows that

$$
\begin{aligned}
\sum_{i=1}^{2} \operatorname{deg}\left(u_{i}\right)+\operatorname{deg}\left(v_{1}\right) & \leqslant(9 s+3)(s-1)+(-2 n+6 s-2)(2 s)+6 s n-18 s^{2}+6 s \\
& =2 s n+3 s^{2}-4 s-3 .
\end{aligned}
$$

Applying (7), we have $\sum_{i=1}^{2} \operatorname{deg}\left(u_{i}\right)+\operatorname{deg}\left(v_{1}\right)<3 s n-\eta_{1} n\left(n-3 \eta_{1} n\right)$, which contradicts (5) because $\varepsilon \ll \eta_{1}$.

By Claim 20, we have $\left|W_{2}\right|=n-3 s<3 \eta_{1} n$. Let $H^{\prime}=H\left[V \backslash W_{2}\right]$. We claim that $\sigma_{2}\left(H^{\prime}\right)>2 n^{2} / 3-\eta_{2} n^{2}$. Indeed, recall that $\operatorname{deg}_{H}(u)+\operatorname{deg}_{H}(v) \geqslant 2 n^{2} / 3-\varepsilon n^{2}$ for any two adjacent vertices $u$ and $v$ of $H^{\prime}$. Since $\left|W_{2}\right|<3 \eta_{1} n$ and $\varepsilon \ll \eta_{1} \ll \eta_{2}$, it follows that

$$
\operatorname{deg}_{H^{\prime}}(u)+\operatorname{deg}_{H^{\prime}}(v) \geqslant 2 n^{2} / 3-\varepsilon n^{2}-2\left|W_{2}\right| n>2 n^{2} / 3-\eta_{2} n^{2} .
$$

Since $\eta_{2} \ll 1$, we may apply Theorem 9 and conclude that either $H^{\prime}$ is a subgraph of $H_{3 s, s}^{2}$ or $H^{\prime}$ contains a perfect matching. In the former case, there is a partition of $V\left(H^{\prime}\right)$ into two sets $|T|=2 s-1$ and $|S|=s+1$ such that for every vertex $u \in S$,

$$
\operatorname{deg}_{H^{\prime}}(u) \leqslant\left(\begin{array}{c}
|T| \\
2
\end{array}\right)=\left(\begin{array}{c}
2 s-1 \\
2
\end{array}\right) \leqslant\left(\begin{array}{c}
2 n / 3-1 \\
2
\end{array}\right)<\frac{2}{9} n^{2} .
$$


On the other hand, since $U \subseteq V\left(H^{\prime}\right)$ and $|U| \geqslant 2 s$, there exists a vertex $u \in U \cap S$ such that

$$
\begin{aligned}
\operatorname{deg}_{H^{\prime}}(u) & \geqslant \operatorname{deg}_{H}(u)-\left|W_{2}\right| n \geqslant s n-\frac{\varepsilon}{2} n^{2}-\left|W_{2}\right| n \\
& \geqslant\left(\frac{n}{3}-\eta_{1} n\right) n-\frac{\varepsilon}{2} n^{2}-3 \eta_{1} n^{2}>\frac{2}{9} n^{2},
\end{aligned}
$$

which is a contradiction. Therefore $H^{\prime}$ must contain a perfect matching, which is a matching of size $s$ in $H$.

Case 2. $\left|M_{1}\right|>3 s-|U|$.

The difference from Case 1 is that, for any edge $e \in M$, we cannot find two disjoint edges $e_{1}, e_{2}$ from $e \cup U_{3} \cup W_{2}$ - otherwise we can replace $M$ by $M \backslash\{e\} \cup\left\{e_{1}, e_{2}\right\}$ contradicting the assumption that $M$ is an optimal matching.

Note that $\left|U_{3}\right|=|U|+\left|M_{1}\right|-3|M| \geqslant 3 s+1-3|M| \geqslant 4$.

Claim 21. For any $e \in M, \sum_{i=1}^{3}\left|L_{u_{i}}\left(e, U_{3} \cup W_{2}\right)\right| \leqslant 3\left(\left|U_{3}\right|+\left|W_{2}\right|+2\right)$.

Proof. Assume $e=\left\{u_{1}^{\prime}, u_{2}^{\prime}, u_{3}^{\prime}\right\} \in M$. For $i=1,2,3$, let $G_{i}$ be the graph obtained from $L_{u_{i}^{\prime}}\left(U_{3} \cup W_{2}\right)$ after adding an isolated vertex $u^{*}$. Then $\left|V\left(G_{i}\right)\right|=\left|U_{3}\right|+\left|W_{2}\right|+1 \geqslant 5$. Since $H$ contains no two disjoint edges $e_{1}, e_{2}$ from $e \cup U_{3} \cup W_{2}$, we know that for any $i \neq j$, every edge of $G_{i}$ intersects every edge of $G_{j}$. The desired inequality thus follows from Lemma 12.

By Claims 17 and 21, we obtain that

$$
\begin{aligned}
\sum_{i=1}^{3} \operatorname{deg}\left(u_{i}\right) & \leqslant 18\left(\begin{array}{c}
|M| \\
2
\end{array}\right)+9|M|+\sum_{i=1}^{3}\left|L_{u_{i}}\left(V(M), U_{3} \cup W_{2}\right)\right| \\
& \leqslant 18\left(\begin{array}{c}
|M| \\
2
\end{array}\right)+9|M|+3|M|\left(\left|U_{3}\right|+\left|W_{2}\right|+2\right) \\
& =(3 n+6)|M| \leqslant 3 s n+6 s .
\end{aligned}
$$

Let $W^{\prime}=\left\{v \in W: \operatorname{deg}(v) \leqslant s n-s^{2} / 2+\gamma^{\prime} n^{2}\right\}$. If $\left|W^{\prime}\right| \leqslant \gamma n$, then we let $H^{\prime}:=$ $H\left[V \backslash W^{\prime}\right]$. By the definition of $W^{\prime}, \operatorname{deg}_{H}(u)>s n-s^{2} / 2+\gamma^{\prime} n^{2}$ for every $u \in V\left(H^{\prime}\right) \cap W$. For any $u \in V\left(H^{\prime}\right) \cap U, \operatorname{deg}_{H}(u)>s n-\varepsilon n^{2} / 2>s n-s^{2} / 2+\gamma^{\prime} n^{2}$ because $s>\tau n$ and $\varepsilon \ll \gamma^{\prime} \ll \tau$. Therefore every vertex $u \in V\left(H^{\prime}\right)$ satisfies

$$
\operatorname{deg}_{H^{\prime}}(u) \geqslant \operatorname{deg}_{H}(u)-n\left|W^{\prime}\right|>s n-\frac{s^{2}}{2}+\gamma^{\prime} n^{2}-\gamma n^{2}>\left(\begin{array}{c}
n-1 \\
2
\end{array}\right)-\left(\begin{array}{c}
n-s \\
2
\end{array}\right)+1,
$$

because $\left|W^{\prime}\right| \leqslant \gamma n, \gamma \ll \gamma^{\prime}$, and $n$ is sufficiently large. By Theorem 1, $H^{\prime}$ contains a matching of size $s$.

We thus assume that $\left|W^{\prime}\right|>\gamma n$ for the rest of the proof. If one of $u_{1}, u_{2}, u_{3}$ is adjacent to a vertex of $W^{\prime}$, then

$$
\sum_{i=1}^{3} \operatorname{deg}\left(u_{i}\right)>4\left(s n-\frac{\varepsilon}{2} n^{2}\right)-\left(s n-\frac{s^{2}}{2}+\gamma^{\prime} n^{2}\right)=3 s n+\frac{s^{2}}{2}-2 \varepsilon n^{2}-\gamma^{\prime} n^{2},
$$


which contradicts (9) because $s>\tau n$ is sufficiently large and $\varepsilon \ll \gamma^{\prime} \ll \tau$.

If none of $u_{1}, u_{2}, u_{3}$ is adjacent to a vertex of $W^{\prime}$, then we distinguish the following two subcases.

Subcase 2.1. $\left|W^{\prime} \cap W_{1}\right|>\gamma n / 2$.

Let $M^{\prime}=\left\{e \in M: e \cap W^{\prime} \neq \emptyset\right\}$, thus $\left|M^{\prime}\right|>\gamma n / 2$. Since $u_{1}, u_{2}, u_{3}$ are not adjacent to any vertex in $W^{\prime} \cap W_{1}$, then for any distinct $e_{1}, e_{2}$ from $M^{\prime}$, we have

$$
\sum_{i=1}^{3}\left|L_{u_{i}}\left(e_{1}, e_{2}\right)\right| \leqslant 12 .
$$

By Claims 17, 21 and (10), we have

$$
\begin{aligned}
\sum_{i=1}^{3} \operatorname{deg}\left(u_{i}\right) & \leqslant\left(18\left(\begin{array}{c}
|M| \\
2
\end{array}\right)-6\left(\begin{array}{c}
\left|M^{\prime}\right| \\
2
\end{array}\right)\right)+9|M|+3|M|(n-3|M|+2) \\
& \leqslant(3 n+6)|M|-6\left(\begin{array}{c}
\left|M^{\prime}\right| \\
2
\end{array}\right) .
\end{aligned}
$$

Since $\left|M^{\prime}\right|>\gamma n / 2$, it follows that

$$
\sum_{i=1}^{3} \operatorname{deg}\left(u_{i}\right) \leqslant(3 n+6)(s-1)-6\left(\begin{array}{c}
\gamma n / 2 \\
2
\end{array}\right),
$$

which contradicts (4) because $s \leqslant n / 3$ and $\varepsilon \ll \gamma$.

Subcase 2.2. $\left|W^{\prime} \cap W_{1}\right| \leqslant \gamma n / 2$.

Since $\left|W^{\prime}\right|>\gamma n$, we have $\left|W^{\prime} \cap W_{2}\right|>\gamma n / 2$. Let $W_{2}^{*}=W_{2} \backslash W^{\prime}$. Then $W_{2} \backslash W_{2}^{*}=W^{\prime} \cap$ $W_{2}$. By Claim 21, we obtain that $\sum_{i=1}^{3}\left|L_{u_{i}}\left(V(M), U_{3} \cup W_{2}^{*}\right)\right| \leqslant 3|M|\left(\left|U_{3}\right|+\left|W_{2}^{*}\right|+2\right)$. Therefore,

$$
\begin{aligned}
\sum_{i=1}^{3} \operatorname{deg}\left(u_{i}\right) & \leqslant 18\left(\begin{array}{c}
|M| \\
2
\end{array}\right)+9|M|+\sum_{i=1}^{3}\left|L_{u_{i}}\left(V(M), U_{3} \cup W_{2}^{*}\right)\right| \\
& \leqslant 18\left(\begin{array}{c}
|M| \\
2
\end{array}\right)+9|M|+3|M|\left(\left|U_{3}\right|+\left|W_{2}^{*}\right|+2\right) \\
& =18\left(\begin{array}{c}
|M| \\
2
\end{array}\right)+9|M|+3|M|\left(\left|U_{3}\right|+\left|W_{2}\right|+2\right)-3|M|\left|W_{2} \backslash W_{2}^{*}\right| \\
& =\left(3 n+6-\frac{3}{2} \gamma n\right)|M|
\end{aligned}
$$

which contradicts (4) because $|M| \leqslant s, \tau n<s$, and $\varepsilon \ll \gamma \ll \tau$. This completes the proof of Theorem 6 .

\section{References}

[1] R. Aharoni and D. Howard, A rainbow r-partite version of the Erdős-Ko-Rado theorem, Combin. Probab. Comput. 26 (2017), 321-337. 
[2] N. Alon, P. Frankl, H. Huang, V. Rödl, A. Ruciński, and B. Sudakov. Large matchings in uniform hypergraphs and the conjectures of Erdös and Samuels, J. Combin. Theory Ser. A 119 (2012), 1200-1215.

[3] P. Erdős, A problem on independent r-tuples, Ann. Univ. Sci. Budapest. Eötvős Sect. Math. 8 (1965), 93-95.

[4] P. Frankl, On the maximum number of edges in a hypergraph with given matching number, Discrete Appl. Math. 216 (2017), 562-581.

[5] H. Hàn, Y. Person, and M. Schacht, On perfect matchings in uniform hypergraphs with large minimum vertex degree, SIAM J. Discrete Math. 23 (2009), 732-748.

[6] J. Han, Perfect matchings in hypergraphs and the Erdős matching conjecture, SIAM J. Discrete Math. 30 (2016), 1351-1357.

[7] I. Khan, Perfect matching in 3-uniform hypergraphs with large vertex degree, SIAM J. Discrete Math. 27 (2013), 1021-1039.

[8] I. Khan, Perfect matchings in 4-uniform hypergraphs, J. Combin. Theory Ser. B 116 (2016), 333-366.

[9] D. Kühn and D. Osthus, Matchings in hypergraphs of large minimum degree, $J$. Graph Theory 51 (2006), 269-280.

[10] D. Kühn, D. Osthus and A. Treglown, Matchings in 3-uniform hypergaphs, J. Combin. Theory Ser. B 103 (2013), 291-305.

[11] T. Łuczak and K. Mieczkowska, On Erdős' extremal problem on matchings in hypergraphs, J. Combin. Theory, Ser. A 124 (2014), 178-194.

[12] K. Markström and A. Ruciński, Perfect matchings (and Hamilton cycles) in hypergraphs with large degrees, European J. Combin. 32 (2011), 677-687.

[13] O. Ore, Note on Hamilton circuits. Amer. Math. Monthly 67 (1960), 55.

[14] O. Pikhurko, Perfect matchings and $K_{4}^{3}$-tilings in hypergraphs of large codegree, Graphs Combin. 24 (2008), 391-404.

[15] V. Rödl and A. Ruciński, Dirac-type questions for hypergraphs - a survey (or more problems for Endre to solve), An Irregular Mind, Bolyai Soc. Math. Studies 21 (2010), $561-590$.

[16] V. Rödl, A. Ruciński, and E. Szemerédi, Perfect matchings in uniform hypergraphs with large minimum degree, European J. Combin. 27 (2006), 1333-1349.

[17] V. Rödl, A. Ruciński, and E. Szemerédi, An approximate Dirac-type theorem for k-uniform hypergraphs, Combinatorica, 28 (2008), 229-260.

[18] V. Rödl, A. Ruciński, and E. Szemerédi, Perfect matchings in large uniform hypergraphs with large minimum collective degree, J. Combin. Theory Ser. A 116 (2009), 613-636.

[19] Y. Tang and G. Yan, An approximate Ore-type result for tight hamilton cycles in uniform hypergraphs, Discrete Math. 340 (2017), 1528-1534. 
[20] A. Treglown and Y. Zhao, Exact minimum degree thresholds for perfect matchings in uniform hypergraphs, J. Combin. Theory Ser. A 119 (2012), 1500-1522.

[21] A. Treglown and Y. Zhao, Exact minimum degree thresholds for perfect matchings in uniform hypergraphs II, J. Combin. Theory Ser. A 120 (2013), 1463-1482.

[22] A. Treglown and Y. Zhao, A note on perfect matchings in uniform hypergraphs, Electron. J. Combin. 23 (2016), \#P1.16.

[23] Y. Zhang and M. Lu, Some Ore-type results for matching and perfect matching in k-uniform hypergraphs, Acta. Math. Sin. - English Ser. 34 (2018) 1795-1803.

[24] Y. Zhang and M. Lu, d-matching in 3-uniform hypergraphs, Discrete Math. 341 (2018), 748-758.

[25] Y. Zhang and M. Lu, Matching in 3-uniform hypergraphs, Discrete Math. 342 (2019), 1731-1737.

[26] Y. Zhang, Y. Zhao and M. Lu, Vertex degree sums for perfect matchings in 3-uniform hypergraphs, Electron. J. Combin. 25 (2018), \#P3.45.

[27] Y. Zhao, Recent advances on dirac-type problems for hypergraphs. In Recent Trends in Combinatorics, volume 159 of the IMA Volumes in Mathematics and its Applications. Springer, New York, 2016. 